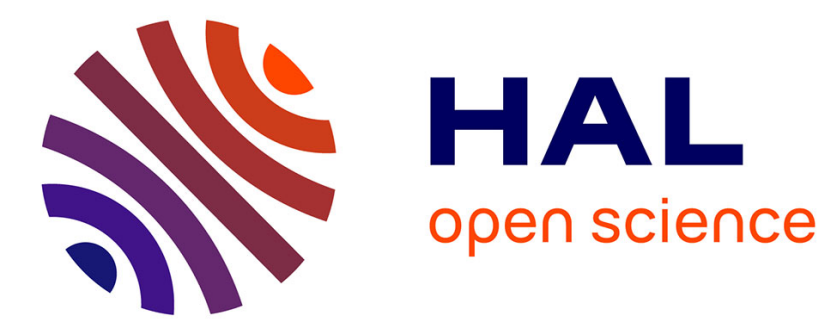

\title{
Périphrases verbales et genèse de la prédication en langue anglaise
}

Didier Bottineau

\section{To cite this version:}

Didier Bottineau. Périphrases verbales et genèse de la prédication en langue anglaise. Colloque: Les Périphrases verbales, Jun 2003, Caen, France. pp.475-495, 10.1075/lis.25.34bot . hal-00012389

\section{HAL Id: hal-00012389 \\ https://hal.science/hal-00012389}

Submitted on 21 Oct 2005

HAL is a multi-disciplinary open access archive for the deposit and dissemination of scientific research documents, whether they are published or not. The documents may come from teaching and research institutions in France or abroad, or from public or private research centers.
L'archive ouverte pluridisciplinaire HAL, est destinée au dépôt et à la diffusion de documents scientifiques de niveau recherche, publiés ou non, émanant des établissements d'enseignement et de recherche français ou étrangers, des laboratoires publics ou privés. 
Didier BOTTINEAU

CNRS - CRISCO « Centre de Recherches Inter-langues sur la Signification en Contexte »

(FRE 2805)

didier.bottineau@crisco.unicaen.fr

\section{Périphrases verbales et genèse de la prédication en langue anglaise}

Recourir à la notion de périphrase verbale, c'est reconnaître à un relateur ou à une locution verbale une unité fonctionnelle qui la rapproche du statut du mot. Ce terme pose ainsi le même problème que celui de partie du discours, mais à un niveau de construction syntaxique plus avancé : il ne s'agit plus d'un mot, mais d'une construction, dont on ne pourrait décider a priori s'il convient d'y voir un syntagme. Pour que la classe des périphrases verbales soit scientifiquement valide, il faudrait idéalement répondre précisément aux questions suivantes : 1) en amont, existe-t-il un profil sémantique fonctionnel qui caractérise la catégorie ? 2) Ce profil se traduit-il par un formatage morpho-syntaxique cohérent qui permette d'envisager l'existence d'une classe formelle ? 3) Cette classe formelle a-t-elle pour fonction, comme les parties de langue, de permettre et prédire des combinatoires, à savoir, de déterminer une pragmatique énonciative et une attente dans la linéarité ? 4) Si cette classe formelle connaît, comme le verbe et le substantif, une valeur par défaut également imposable à des notions non affines, peut-on classer sémantiquement les périphrases verbales en fonction de l'homogénéité ou du caractère marqué du rapport forme / sens qui les sous-tend ? La réponse à ces questions contribuerait à livrer le mode d'emploi contractuel de la catégorie mis en œuvre dans l'interlocution et fonderait l'existence même de la classe sur la démarche qui la sous-tend. L'approche est naturaliste, fonctionnelle et sémasiologique ; elle suppose donc que la question soit appliquée à une langue à la fois, puis plusieurs dans une approche contrastive et typologique, enfin aux langues en général pour sonder la pertinence d'hypothétiques généralisations. Les lignes qui suivent se concentrent donc sur ce que la prise en compte de la structure des périphrases verbales de l'anglais permet d'apporter à la problématique qui précède.

\section{La périphrase verbale entre la langue et le discours}

La psychomécanique du langage, modèle linguistique constructiviste fondé par Gustave Guillaume, se concentre sur la modélisation des systèmes mentaux organisés mis en œuvre par l'énonciateur détenteur de la faculté de langage (nommée « langue ») et en instance d'acte de langage (ou « discours »), ce qui en fait une linguistique cognitive essentiellement binaire. Ce binarisme permet d'envisager pour toute catégorie lexicale deux états fondamentaux, successifs et complémentaires : l'état puissanciel de la partie de langue virtuelle envisagée hors emploi, et l'état effectif ou actualisé mis en œuvre par l'énonciation dans la transition langue / discours. Le substantif connaît ainsi un état puissanciel (hors détermination) et un état effectif (le substantif avec sa détermination, ou syntagme nominal). La procédure de construction du syntagme effectif et la configuration qui en résulte sont régies par les propriétés constitutives de la catégorie de langue lui servant de départ, les «formes vectrices ». Par contraste, la périphrase verbale complexe, formée d'un auxiliaire et d'un « préverbe » (is to), n'est pas un mot de langue isolable en tant que catégorie et, partant, ne connaît que des états actualisés relevant du discours mais non dérivables d'une primitive puissancielle, ce qui l'oppose notamment aux auxiliaires modaux, opérateurs de langue formés d'un mot unique dont la catégorisation ne dépend pas du contexte ; mais on ne peut 
pas non plus les considérer comme des structures discursives improvisées et dénuées de tout principe de préconstruction. Admettre leur existence en leur prêtant un statut cognitif stabilisé chez l'énonciateur revient donc à relativiser une lecture radicale du binarisme guillaumien en la nuançant par une approche stratifiée interposant entre les extrêmes des plans de puissance et d'effet un champ de l'effection ou actualisation en lequel plusieurs degrés de construction sont envisageables. Cette démarche, contenue en germe par certains systèmes ternaires proposés par Guillaume tels que la chronogenèse (avec ses trois chronothèses du mode quasinominal, du subjonctif et de l'indicatif) ${ }^{1}$, est appliquée par Joly et Roulland ${ }^{2}$ à la modélisation de l'énonciation sous le terme d'effection et par Valin (1981) à celle de la syntaxe génétique sous le terme de logogenèse. Elle est radicalisée par la sémantique de Pottier (1992), qui réfute le binarisme du tenseur binaire radical au profit du ternarisme du trimorphe. Quelque position que l'on adopte dans ce débat, la notion de périphrase verbale s'inscrit dans le cadre d'une reconnaissance au moins locale du ternarisme cognitif dans la construction des systèmes grammaticaux : sans être préconstruite comme un mot de langue ni improvisée comme un énoncé de discours, la périphrase verbale se construit entre les deux, en logogenèse, et ne peut non plus être ramenée à l'état actualisé d'une partie de langue, c'est à dire qu'elle n'a pas valeur de syntagme, mais de relateur prédicationnel entre sujet et prédicat.

\section{Do et la « forme simple »}

En observation des phénomènes de surface, on sait que l'anglais admet une structure de prédication assertive simple non auxiliée de forme sujet + verbe $\mathrm{S}+\mathrm{V}(-\mathrm{s} /$-ed $)$, I smoke cigarettes, mais que celle-ci est incompatible avec tout marqueur morphologique, syntaxique ou prosodique de nature à relativiser ou neutraliser la visée assertive de l'énoncé. Le morphème not est inapplicable au couple $\{\mathrm{S}+\mathrm{V}\}:{ }^{*}$ I not smoke cigarettes $>$ I do not smoke cigarettes. Le syntaxème [INV] (pour inversion syntaxique) aussi : *Smoke you cigarettes? > Do you smoke cigarettes? Le prosodème [LOW RISE], élévation du ton bas au ton moyen appliqué à l'accent d'intensité principal de l'énoncé (main prominence) porté par le noyau (nucleus), généralement la dernière syllabe accentuée de la proposition (ici -rette), est porteur en cette position finale d'une indication de non-validation prédicationnelle par l'énonciateur, conférant à l'ensemble une valeur interrogative avec sollicitation du co-énonciateur pour la prise en charge ou non de la validation : *You smoke cigarettes ? > Do you smoke cigarettes ? (au sens interrogatif de Est-ce que vous fumez des cigarettes?; par contre, s'il s'agit d'interroger par le prosodème un constat dont la validité est admise par le couple $\mathrm{S}+\mathrm{V}$, alors cette construction est couramment attestée: Ah bon, / Tiens, vous fumez des cigarettes?) Enfin, la présence d'une modalité anti-assertive dans le contexte avant, de type négatif ou hypothétique, bloque l'assertion simple par $\{\mathrm{S}+\mathrm{V}\}$ et appelle do dit emphatique à valeur polémique (il vaudrait mieux parler de dialogique) : You don't remember what I said. - Yes, I *remember / > do remember what you said.

Le constat de l'incompatibilité entre $\{\mathrm{S}+\mathrm{V}\}$ assertif et toute marque de réfutation dialogique amène à poser que $d o$ opère une assertion inscrite dans un environnement dialogique défavorable alors que $\{\mathrm{S}+\mathrm{V}\}$ monologique (ou non explicitement dialogique) se contente de constater la validité référentielle du procès posé par simple contiguïté $: \mathrm{S}+\mathrm{V}$ déclare, $\mathrm{S}+$ do $+\mathrm{V}$ répond ${ }^{3}$, soit à une sollicitation extérieure au locuteur par un autre locuteur antérieur (cas de la réponse à une question effectivement posée ou de la riposte polémique), soit à une

\footnotetext{
${ }^{1}$ Guillaume 1929.

2 Joly 1987, 22.

${ }^{3}$ Plusieurs modèles réfutent la pertinence de la frontière séparant la grammaticalisation de la pragmatique (Douay 2000) et postulent au contraire que la fonction des systèmes est de grammaticaliser la topologie des configurations des rapports allocutifs et dialogiques.
} 
sollicitation au seul locuteur considéré (ce qui revient à envisager la structure comme la marque polyphonique d'une pluralité d'énonciateurs). Dans le cas de la question Do you smoke cigarettes ? do répond au blocage du constat positif par $\{\mathrm{S}+\mathrm{V}\}$ induit par le prosodème ascendant en restaurant la prise en charge de l'opération de soudure prédicationnelle une fois que celle-ci a été compromise en première instance par un facteur adverse.

Dans le cas de la négation ${ }^{4}$, c'est not qui inhibe la fonction de soudure de $\{\mathrm{S}+\mathrm{V}\}$, laquelle est réparée par do selon un mécanisme de suspension et de déblocage : à l'intérieur même de la structure et chez un locuteur unique, des programmes cognitifs adverses, positifs et négatifs en regard de la prédication, sont mis en conflit et la dicibilité même de la relation prédicative passe par une résolution de l'antagonisme. Le morphème not lui-même se laisse analyser en ces termes : la négation no, en rejetant la validation de la mise en rapport d'un couple $\{\mathrm{S}+\mathrm{V}\}$, rend la prédication en question inénonçable (*No I smoke cigarettes, *I no smoke cigarettes) et il faut suspendre l'effet inhibiteur de no sur la prédication en lui adjoignant un morphème $t^{5}$, livrant not, pour pouvoir procéder au rétablissement de la soudure prédicationnelle do not / don't, laquelle est, sans cela, irréalisable (*No I do smoke cigarettes, *I do no smoke

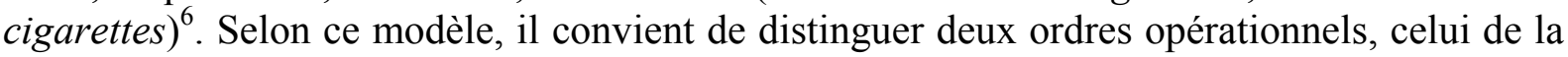
construction de la prédication d'une part, avec son blocage (par un morphème, un syntaxème ou un prosodème) et son déblocage (par $d o$ ); et d'autre part celui de son énonciation, la lecture linéaire du schème prédicationnel postérieurement à sa construction, avec la marque de déblocage do qui, toujours, précède celle de blocage (not etc): à l'intention de l'allocutaire, le locuteur commence par énoncer la résolution du problème avant d'en rappeler les termes.

Dans la systématique énonciative, évolution du modèle guillaumien développée par Joly (1987), ces deux ordres correspondent à la syntaxe génétique pour le premier et à la syntaxe résultative pour le second. Selon le modèle guillaumien, un énoncé linéaire porteur d'une relation prédicative est le produit sémiologique énoncé résultant d'une démarche cognitive constructionnelle. Celle-ci s'opère en deux temps: 1) le locuteur se donne un projet de relation prédicative ou proposée de discours, à savoir un couple sujet / prédicat entre lesquels il envisage de valider une relation repérée par rapport à l'instant de parole ${ }^{7}$. Le couple en question correspond ici à $<$ you $>$ et $<$ shoot this horse $>$. 2) le locuteur soumet la proposée de discours à un regard évaluateur pondérant le degré de conformité de la notion de procès considérée au référent extralinguistique visé. En cas d'accord entre le procès exprimé par la proposée de discours et le référent visé, le couple sujet / prédicat est actualisé en l'état sans

\footnotetext{
${ }^{4}$ Bottineau 2004b.

${ }^{5}$ Dans le cadre de la théorie des cognèmes, le submorphème $-t$ des grammèmes est analysé comme une marque de passé métalinguistique de préconstruction d'une relation. Not renvoie au vécu cognitif du locuteur une négation antérieurement mise en place par no, ou présupposée. Parallèlement, yet concessif préconstruit l'approbation que $-s$ de yes présentifie dans le dialogue : yes = j'approuve (maintenant), yet = j'ai déjà approuvé (avant), ce qui me permet à présent d'envisager de récuser (= la concession). Cette alternance $s / t$ se retrouve dans d'autres système tels que les flexions verbales (has / had) et les démonstratifs (this de définition, that de prédéfinition): Bottineau 2004c. Selon Delmas 1987 le rôle central de la morphologie grammaticale est de signaler les écarts entre ordre constructionnel et énonciation linéaire, ce qui fait de $-t$ un marqueur du temps de l'expérience cognitive, le temps opératif de Guillaume.

${ }^{6}$ Le cas de I have no cigarettes left est différent: no n'y bloque pas la prédication, mais la détermination nominale. Celle-ci n'étant pas le pivot indispensable de la connexion sujet / prédicat, elle n'a pas obligatoirement à être restaurée par une forme substitutive de type auxiliaire. Si le mécanisme de préconstruction no $+t>n o t$ est appliqué, la détermination nominale peut réapparaître : not any. La combinaison no $+\mathrm{N}$ rejette la sélection lexicale visée (par opposition au reste du paradigme envisageable) alors que la combinaison not + any porte sur la quantification du référent sans l'envisager dans le cadre d'une telle opposition: there is no sugar left (implique qu'il manque du sucre, mais pas des autres ingrédients); there isn't any sugar left (quantification non contrastive : le sucre était déjà posé comme topique).

${ }^{7}$ Contrairement au schéma de lexis de Culioli, la proposée de discours est orientée du sujet vers le prédicat : du syntagme recevant le « fichier » que l'on ouvre vers celui recevant la modification de son contenu.
} 
aucune discussion de la relation prédicative elle-même, laquelle n'est grammaticalisée par aucune marque auxiliée explicite. On obtient alors une transformée de discours dont la morphosyntaxe est généralement conforme à celle des éléments séparés formés par le protosujet et le proto-prédicat antérieurement construits au niveau de la proposée de discours : They shoot horses, don't they?( « On abat bien les chevaux »).

Ceci revient à dire que la stratégie de l'anglais est, paradoxalement, de ne pas prédiquer en cas d'assertion simple non explicitement inscrite dans le rapport dialogique : le locuteur livre à l'allocutaire le couple sujet / prédicat en «kit à construire » en vue de lui laisser le soin de calculer en sémantique interprétative ce qu'implique leur mise en rapport sans avoir de luimême réalisé la connexion; ce couple est énoncé par des mots un instant avant que ne se réalise sa soudure, non marquée en raison de son caractère non problématique ${ }^{8}$. Cette délégation de la jonction prédicationnelle à l'allocutaire se manifeste en anglais par divers faits tels que l'absence d'accord en rang de personne ${ }^{9}$ et la non-restriction de la portée référencielle du procès si ce n'est par la connaissance des propriété des entités impliquées ${ }^{10}$. Pour le rôle de la périphrase verbale dans l'économie générale du système du verbe anglais, cela signifie que 1) le locuteur ne recourt pas à son utilisation lorsqu'il se contente de livrer à l'allocutaire le couple $\mathrm{S}+\mathrm{P}$ dont la relation reste à valider par l'allocutaire avec calcul maximalement ouvert de la portée référentielle, et, corollairement, que 2) le locuteur recourt à une périphrase verbale en vue d'expliciter les paramètres qui président à la validation de la connexion, avec des effets de sens restreints, contraints et contextuellement motivés.

\section{Typologie cognitive des périphrases verbales de l'anglais : modalisation primaire et modalisation secondaire}

On réservera dans cette étude l'emploi du terme de modalisation de la relation prédicative à une valeur très spécifique : n'est pas modalisée une prédication dont la soudure n'est pas prise en charge par le locuteur, mais déléguée à l'allocutaire, ce qui se traduit par l'absence de marque de relation auxiliée interposée entre le sujet et le verbe (présent simple et prétérit en assertion non dialogique); est modalisée une prédication dont la soudure est effectivement prise en charge par le locuteur et marquée par un auxiliaire spécifiant les conditions de la connexion. En poursuivant notre démarche sémasiologique, à savoir la modélisation des faits de structuration cognitive sur la base de l'observation des faits de morphosyntaxe ${ }^{11}$, on distingue deux classes de configurations : celles formées au moins d'un auxiliaire et d'une base verbale non fléchie, avec ou sans to (he is to come at five, he must help us, he does speak Japanese); et celles formées d'un auxiliaire et d'une base verbale fléchie (-ing, participe passé).

Le verbe connaît donc trois traitements morphologiques successifs hiérarchisés comme suit : 1) si la base verbale est précédée de $t o$, d'un modal ou de $d o$, toute flexion est proscrite. 2) si

\footnotetext{
${ }^{8}$ Douay 2000, 116.

${ }^{9}$ Le $-S$ de «troisième personne du singulier » (A dog barks) est en réalité une marque de pluralisation du référent du prédicat verbal exprimant l'événement : on envisage toutes les occurrences d'aboiement associables à un individu canin donné (Bottineau 2004c). Ce $-s$ disparaît si le sujet est lui-même pluriel (Dogs bark), la pluralisation du référent du prédicat étant impliquée par sa connexion à un sujet à référent multiple.

${ }^{10}$ En raison de l'absence de filtrage par un auxiliaire, le présent simple exprime un procès qui sera appliqué à tout référent potentiellement concerné tant pour le sujet que pour le prédicat, d'où sa propension à exprimer des faits généraux (oil floats on water), sauf lorsque des contraintes sémantiques particulières confinent la référence à une occurrence unique (the Queen walks to the throne, I pour the milk into the saucepan), auquel cas l'effet d'expansion référencielle se traduit par une vision filmique de l'événement dont tous les instants successifs sont validés (perfectivité).

${ }^{11}$ Par opposition à une démarche onomasiologique comme la sémantique de Pottier (1992), qui classe les faits de langue en fonction d'un schème cognitif intégrant postulé, le trimorphe.
} 
la base verbale n'est pas séparée du sujet (si la connexion est déléguée à l'interprétation), une flexion est possible ( $-s$ de d'actualisation référencielle, -ed de virtualisation référencielle). 3) si la base verbale est précédée de be ou have, une flexion est obligatoire (be + -ing, be / have + participe passé).

Il apparaît ainsi que la prédication directe non auxiliée (cas 2) constitue la position intermédiaire, le pivot central du système : elle saisit par des marques non stabilisées l'instant critique en lequel la validation de la connexion se négocie entre les partenaires de l'interlocution avec la démission du locuteur et la participation différée de l'allocutaire.

Ce pivot est précédé de l'ensemble des structures qui proscrivent toute flexion sur le verbe (cas 1) : to, les modaux et do apparaissent lorsque l'énonciateur s'abstient définitivement de valider la connexion et délègue intégralement à l'allocutaire la responsabilité de ratifier le procès proposé. Dans it must be true, must bloque be en phase non fléchie : le modal a interrompu le processus de validation du rapport it / be true, qui, sans cette interception, prendrait la forme it is true.

Enfin, le pivot central est suivi de l'ensemble des structures qui nécessitent une flexion: l'auxiliaire be déclare l'existence d'un rapport encore valide $(+-i n g)$ ou périmé $(+$ participe passé), l'auxiliaire have rapporte à un sujet un rapport prédicationnel périmé (+ participe passé). Be et have ont en commun de déclarer la soudure prédicationnelle comme déjà validée à l'instant où des morphèmes grammaticaux en saisissent l'état résiduel (persistance pour be, décadence pour have) et de renvoyer au passé cognitif des colocuteurs la validation ellemême, ce que l'on nomme préconstruction ${ }^{12}$.

Les périphrases verbales sont ainsi distribuées de part et d'autres de l'assertion directe selon une chronologie cognitive : to, les modaux et $d o$ marquent la connexion avant l'instant de sa validation et impliquent que le locuteur en propose le principe (to), milite en sa faveur ou en pondère le degré d'avancement (modal), voire fait maximalement pression sur l'allocutaire pour qu'il en admette la faisabilité $(d o)$, mais dans les trois cas, le locuteur se heurte à une résistance l'amenant à renoncer à déléguer cette validation sans mot dire et à envisager l'existence d'une distance séparant son regard présent de la validation de la connexion (le pivot central de l'assertion directe). Cette première classe de périphrases relève de la modalisation primaire: marquage de la prédication par le locuteur en un état antérieur à sa validation et calcul du parcours non effectué. Inversement, les périphrases en be et have dénotent 1) l'étendue du recul pris à l'instant de parole par le locuteur par rapport à la validation de la prédication (permanence pour -ing, révocation pour -ed) et 2) la nature du rapport entre sujet et procès à l'instant de parole (conjonction pour be, disjonction pour have). Cette seconde classe de périphrases relève de la modalisation secondaire: marquage de la prédication par le locuteur en un état résultant postérieur à sa validation, avec évaluation du recul impliqué.

Un élément que l'on ne peut développer ici en détail est que le système des morphèmes verbaux reflète celui des prépositions spatiales tant par la morphologie que par le sens : 1) pour les flexions, to préverbal («à accomplir ») reproduit to (directif), -ing (accomplissement = intériorité aspectuelle) reproduit in (intériorité spatiale), -ed (résultat d'un procès) reproduit at (locatif $=$ résultat d'un mouvement spatial). 2) pour les auxiliaires, do (prospection temporelle) actualise to (direction), be (introspection temporelle) actualise in (intériorité

\footnotetext{
${ }^{12}$ On distingue 1) préconstruction : relation dont la validation est déclarée antérieure à l'instant de son marquage morphologique ; 2) présupposition: ensemble des connaissances considérées comme partagées à l'instant de parole ; 3) anaphore : procédure de reprise d'un élément déjà énoncé par un marqueur spécifique, comme th- en anglais ; 4) thématisation : installation d'un syntagme en position « gauche » de l'énoncé (aperturale en séquence énonciative linéaire), au contact de l'avant texte (fait d'iconicité syntaxique) et signifiant la poursuite d'un thème discursif acquis sans solution de continuité (par opposition à l'anaphore, laquelle restaure un thème momentanément abandonné).
} 
spatiale), have (rétrospection temporelle) actualise at (présupposition de mouvement spatial). Par l'étude des relations d'isomorphisme / isosémisme de ces trois système, la théorie des cognèmes ${ }^{13}$ en a montré l'homologie de structuration cognitive : to / in / at intègre un schème vocalique $/ \mathrm{u} /-/ \mathrm{i} /-/ \mathfrak{a} /$ que développe $d o /$ be / have et un schème consonantique $t-/-n /-t$ que développe to / -ing / -ed, ces deux derniers systèmes réanalysant séparément les deux schèmes constitutifs du premier.

Le système global de la modalisation est donc ternaire: 1) modalisation primaire = périphrases verbales en to / MOD / $d o+$ base verbale ; 2) modalisation nulle $=\mathrm{S}+\mathrm{V}(-s /-e d)$; 3) modalisation secondaire $=b e+\mathrm{V}$-ing/-ed, have $+\mathrm{V}$-ed. Le schéma suivant illustre cette distribution et inclut les constructions à sujet régi (Bottineau 1999).

${ }^{13}$ Bottineau 2002, 2003. 


\section{Typologie des modalisations de la relation prédicative en syntaxe génétique}

1. MODALISATION PRIMAIRE de l'idée regardée par l'idée regardante : prédication à venir

A. analytique : to $=$ relation prédicative in posse (en puissance)

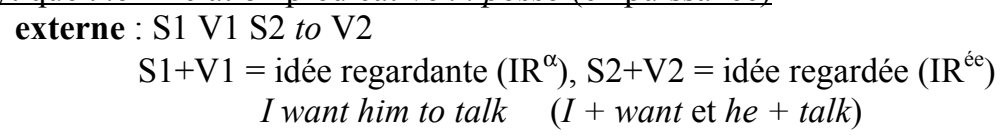

1) $\mathrm{S}+\mathrm{V} 2=\mathrm{IR}^{\mathrm{e} e}, \mathrm{~V} 1=\mathrm{IR}^{\alpha}$ relatorisée sans sujet He began to talk $\quad(\mathrm{He}+$ talk et begin $)$

2) $\mathrm{S}+\mathrm{V} 2=\mathrm{IR}^{\mathrm{e} e}, \mathrm{~V} 1=\mathrm{IR}^{\alpha}$ à sujet coréférentiel He wanted to talk (He + talk et he + want)

B. synthétique : modal : relation prédicative in fieri (en devenir)

$\mathrm{S}+\mathrm{V}=\mathrm{IR}^{\mathrm{e} e}$, modal $=\mathrm{IR}^{\alpha}$ relatorisée He will talk

2. PAS DE MODALISATION de l'idée regardée par l'idée regardante = prédication présente simple et actualisée sans auxiliaire ni rection : présent, prétérit (sans do ni did). RP in esse (actuelle)

$\mathrm{S}+\mathrm{V}=\mathrm{IR}^{\mathrm{e} e}, \mathrm{IR}^{\alpha}=\varnothing:$ He talks / talked

(pas d'interception ni de discussion des conditions d'actualisation)

3. MODALISATION SECONDAIRE : relation prédicative dépassée et virtualisée

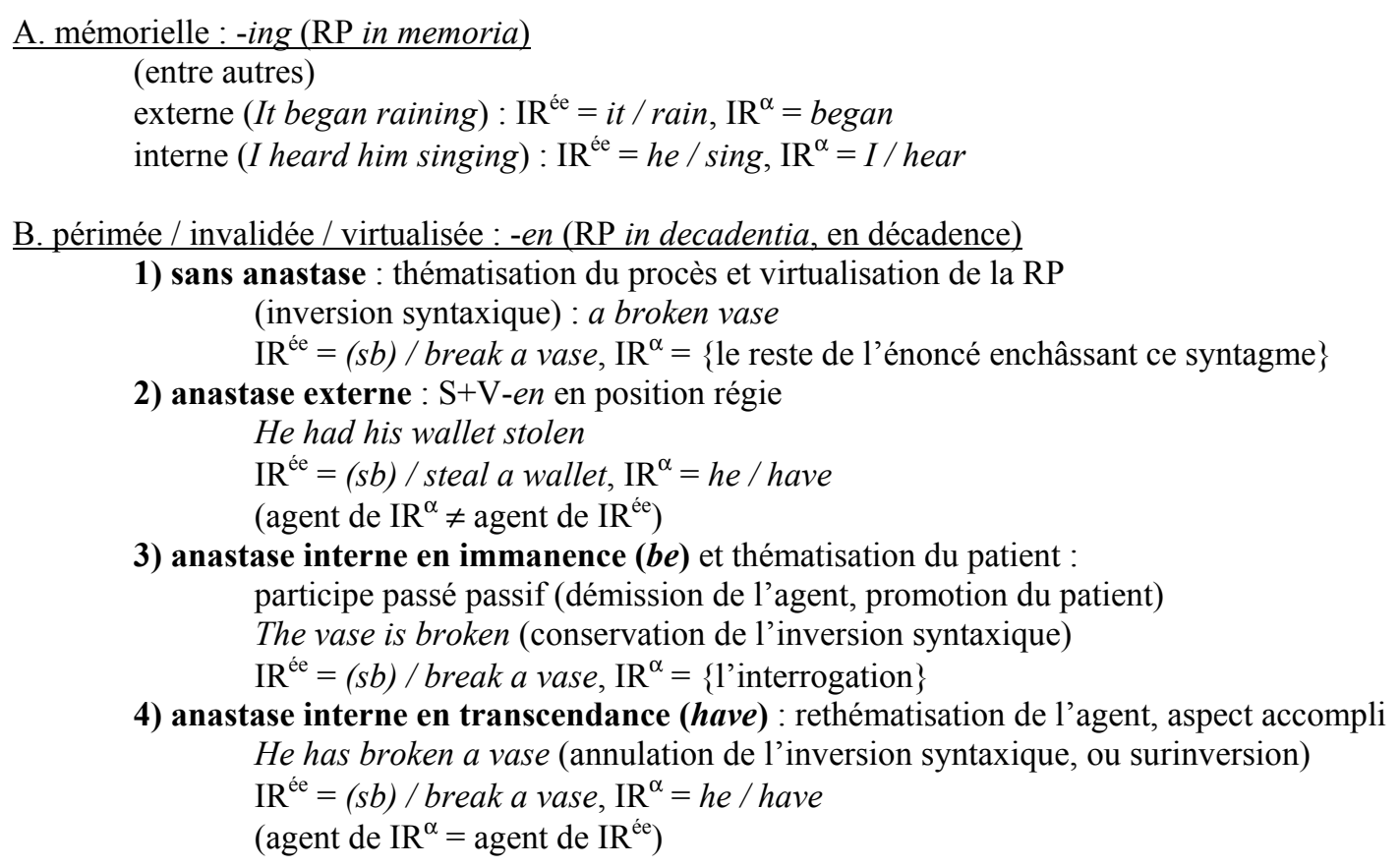

Cette topologie coïncide avec les prédictions du modèle pottiérien mais son obtention se fait par voie sémasiologique et non onomasiologique: il y a convergence sur la base de présupposés et méthodes adversatifs. Toutefois elle coïncide aussi avec la topologie du tenseur binaire radical guillaumien: la modalisation primaire instancie l'avant de la prédication dont elle vise l'actualisation et saisit les étapes puissancielles sur un continuum ; 
la modalisation secondaire instancie l'après de la prédication dont elle préconstruit la validation et saisit les réalisations effectives. Le pivot central de la modalisation nulle par l'assertion directe non auxiliée instancie le seuil critique séparateur des deux tensions.

Cette ambivalence du binaire et du ternaire est emblématique du conflit opposant Pottier et Guillaume : Guillaume privilégie les deux tensions, Pottier décrit les trois positions qu'elles déterminent, et tout psychosystème, comme la chronogenèse, est susceptible de paraître binaire ou ternaire selon le point de vue adopté. Le schème ici proposé converge également à bien des égards avec diverses propositions de Culioli (analyse de to) et d'Adamczweski (la préconstruction) mais il s'en distingue fortement en positionnant zéro comme centre pivotal. Ceci rejoint, en dernier ressort, le modèle Douay / Roulland ${ }^{14}$, lequel voit dans les marqueurs grammaticaux la spécification des configurations du rapport dialogique : l'absence de marque correspond au rapport interlocutif direct (R.I.D) avec une prédication non problématique qu'il ne convient guère de marquer; la modalisation primaire grammaticalise un accord allocutif préalable minimal sur le projet prédicationnel suspendu et mis en question (configuration 1), avec association de l'allocutaire; la configuration 2, dissociative, sature la procédure de construction prédicationnelle, avec un locuteur qui impose à l'allocutaire un positionnement définitif qu'il ne s'agit plus que d'interpréter sans renégociation.

\section{La modalisation primaire}

La modalisation primaire, premier versant du schème des trois niveaux constructionnels de la prédication, se partage elle-même en trois sous-systèmes, reproduisant par mise en abîme l'organigramme intégrant: $\mathrm{AUX} / \mathrm{V}+$ to $+\mathrm{BV}, \mathrm{MOD}+\mathrm{BV}$, do $+\mathrm{BV}$.

\subsection{To}

Dans le système des prépositions spatiales, to, à partir d'un repère explicite ou implicite, cible une position au sein d'un paradigme de possibles pertinents en contexte (to London vs to + toute autre destination implicitement exclue) : to figure le parcours mental possible ou virtuel par laquel un animé humain pourrait atteindre cette cible, sans que cela n'implique une prévision de passage à l'acte ; la représentation fictive du mouvement ne vaut pas tant pour elle-même qu'en tant que moyen de sélection d'une cible figurée comme destination possible $^{15}$, qui peut devenir destination effective en présence d'un verbe de mouvement comme go, d'attribution comme give, etc. : go évoque un mouvement physique attribuable à un expérienceur percevable, ou assumé tel, et relève d'une sémantique lexicale à orientation référencielle et extérieure aux supports cognitifs de l'acte de langage que sont les partenaires de l'interlocution; to véhicule un mouvement psychique inobservable, interne à la dynamique cognitive, permettant la construction de la relation de positionnement voulue : to relève d'une sémantique grammaticale à valeur métalinguistique. La valeur fondamentale de to préverbal est conforme à celle de la préposition, seul le domaine d'application change ${ }^{16}$ : dans he decided to stay at home, to figure un mouvement non référenciel orienté vers stay at home en vue de cibler ce prédicat $^{17}$, ce qui signifie non pas promettre son avènement, mais le poser

\footnotetext{
${ }^{14}$ Douay 2000, 114-120.

${ }^{15}$ Par contraste, towards implique que la cible spatiale introduite n'est pas la destination du mouvement exprimé par le verbe, d'où la possibilité qu'il s'agisse d'une zone étendue ou vague : towards the north.

${ }^{16}$ Il est donc peut-être inutile de complexifier l'analyse en recourant à la métaphorisation ou la théorie guillaumienne de la dématérialisation et de la subduction.

17 La préposition to se grammaticalise en préverbe lorsque le paradigme de destinations géographiques «visables » est remplacé par un paradigme de prédicats eux aussi «visables ». La «forme schématique » (Culioli) ou le « mouvement abstrait» (Langacker) sont préservés, mais elle instancie des « espaces mentaux »
} 
comme option retenue par le locuteur par opposition au paradigme des autres options paradigmatiquement pertinente en contexte ${ }^{18}$, comme par exemple go shopping ${ }^{19}$. A ce niveau précoce de la syntaxe génétique, le locuteur ne s'intéresse pas encore à la sélection de la notion devant instancier le sujet ${ }^{20}$, ce qui met to dans l'incapacité de valider à lui seul une soudure prédicationnelle entre un prédicat en cours de sélection et un sujet non présélectionné, *to stay at home. Il n'est donc pas possible, dans un même énoncé, de marquer à la fois la sélection du prédicat par to et la validation de sa connexion au sujet par do: *John does / did to stay at home. Avec la mise en discussion de la validation du lien prédicationnel, do occulte mécaniquement le sélecteur to parce que celui-ci renvoie à une étape cognitive révolue : John does / did stay at home. To marque ainsi l'état puissanciel de la prédication ; or, étant donné qu'un énoncé, pour être énonçable, doit articuler un sujet à un prédicat par une connexion validée, il ne peut jouer ce rôle et marquera inévitablement une prédication secondaire et régie par un verbe enchâssant.

Plusieurs configurations sémantiques satisfont à cette exigence :

1) le procès n'est que partiellement actualisé par un verbe aspectuel qui prélève une fraction de l'événement (begin, start, continue, cease) en remplacement de do qui en couvrirait la totalité : it began to rain $(* \text { it did to rain })^{21}$.

2) la sélection du prédicat par le locuteur, marquée par to, est redoublée par un verbe lexical signalant que le référent du sujet grammatical aussi réalise le choix psychologique de viser ce même procès, ce qui suppose que le verbe modalisateur impute au sujet un regard et une activité mentale (wish, want, expect, decide, promise): he decided to stay at home. Le prédicat (stay at home) choisi (to) par le locuteur coïncide avec le principe d'action (stay at home) retenu par l'agent (decided) : la visée grammaticale issue du locuteur (to) se superpose à une visée lexicale rapportée au sujet par le verbe psychologique. Le locuteur relaie le choix rapporté au sujet grammatical, s'effaçant au profit ce dernier.

3) les périphrases verbales en be et have, contrairement aux verbes psychologiques, signalent que la visée lexicale préexiste au référent du sujet lui-même : he is to stay = he is supposed / expected (by somebody else) to stay at home. A la différence du verbe psychologique actif qui pose le référent du sujet comme source agentive de la visée lexicale, le verbe psychologique au passif pose le référent du sujet comme cible et patient de la visée lexicale, ce qui augmente d'un cran l'effet de polyphonie en invoquant une tierce personne humaine comme source initiale de la visée, distincte et du locuteur, et du sujet : he is expected to stay at home < one expects him to stay at home.

La périphrase verbale en be + to grammaticalise cette configuration en occultant la spécification du type de modalité lexicale qui préside à la sélection du prédicat : le locuteur justifie le choix du prédicat qu'il réalise (to) par un choix préexistant au sujet $(i s)$ et rapporté à une tierce personne. Have prolonge cette procédure en faisant de cette sélection acquise issue d'une tierce personne une propriété du référent sujet : he has to stay at home; l'implication

différenciés (Fontanille): l'environnement spatial (préposition) vs l'environnement discursif (= le choix d'un prédicat se fait dans l'ensemble des rhèmes possibles à l'instant considéré).

${ }^{18}$ Cette analyse coïncide avec celle d'Adamczewski, mais diffère de celle de Culioli, pour qui le paradigme se réduit à l'opposition entre une option (to stay at home) et son contraire (not to stay at home).

${ }^{19}$ De ce fait to est compatible avec un regard rétrospectif excluant la futurité dès lors que c'est bien le choix de l'événement modalisé qui est en jeu, et on peut retenir la notion de ciblage ou de visée dans ce sens strict, dégagé de toute corrélation aspectuelle : I was glad to see you.

${ }^{20} \mathrm{Il}$ y a donc quatre traitements possibles pour le sujet d'infinitif (Bottineau 2001) : 1) le site n'est pas instancié (To be or not to be $)$ 2) to récupère sur sa gauche un sujet puissanciel que lui fournit l'objet du verbe recteur (I expect you to succeed) ; 3) la préposition dative for anticipe sur l'attribution du prédicat à un sujet à venir (For him to succeed would be surprising) ; 4) en cas de présence d'un sujet effectif, la prédication est rompue par une pause marquée par une virgule (A gentleman, to treat a woman thus!).

${ }^{21}$ Pour une étude du système des verbes aspectuels régissant une forme verbale non finie, cf. Bottineau $2002 \mathrm{~b}$. 
sémantique est l'inévitabilité de l'actualisation de l'événement correspondant au prédicat visé, par opposition à $b e+t o$, qui n'annonce aucunement la réalisation effective. Ce contraste instancie une occurrence du schème Douay / Roulland, avec be + to en configuration 1 (le locuteur pose un programme mais s'abstient de prédire sa réalisation, laissant à l'allocutaire la marge de manœuvre nécessaire pour qu'il puisse se faire une opinion librement) et have + to en configuration 2 (le locuteur dépasse le programme et impose le choix d'une option, l'allocutaire n'ayant plus qu'à souscrire). Dans la structure en have to, c'est une tierce personne et elle seule qui est à l'origine du programme comportemental visé et attribué au référent du sujet, dont la responsabilité est de facto exclue. Ceci explique l'existence d'une troisième périphrase, have + got + to, qui interpole le participe passé du verbe lexical «obtenir » en vue de focaliser une part de responsabilité du référent agentif dans la détermination du programme qu'il lui incombe de réaliser : I've got to go shopping (Ayant laissé la réserve de nourriture s'épuiser, je me trouve dans l'obligation d'aller faire les courses).

Il existe donc trois configurations compatibles avec to : 1) le prélèvement d'un fragment du procès par un verbe aspectuel ; 2) la réattribution de la source de la visée au sujet grammatical par un verbe psychologique ; 3) la réattribution de la source de la visée à une tierce personne autre que le sujet grammatical par un verbe psychologique passif (be expected to) ou l'expression d'un jugement (be apt to, inclined to, likely to) de tendance, de probabilité dont le locuteur ne se pose pas comme l'auteur exclusif. Les périphrases non lexicales implicitent la nature de la visée modale. Elles ont souvent été analysées comme non modales en ce que le locuteur n'assume pas l'exclusivité de sa responsabilité dans le jugement. En réalité, notre analyse suggère que ces périphrases fragmentent la modalité en rapportant différenciellement au locuteur le ciblage du procès visé et à une autre personne humaine (le sujet ou une tierce personne) la détermination du type de jugement modal qui a présidé à la sélection: les périphrases en to réalisent une modalisation analytique et distribuée entre plusieurs sources.

\subsection{Les auxiliaires modaux (may, can, must, shall, will)}

L'auxiliaire modal anglais est une pure marque de mise en relation prédicative qui s'interpose iconiquement entre le sujet et le prédicat (he will come). Ne pouvant être incorporé au prédicat, il récuse tous les traitements morphosyntaxiques dévolus à ce dernier (*to + MOD, *AUX + MOD : *can will, MOD-s: *he musts, *MOD-ing: *maying): sa fonction de relateur le contraint à ne posséder que des formes temporellement repérées par rapport à l'instant de parole, donc présentes ou passées dans le système binaire de l'anglais (may, $m i g h t)^{22}$. Le modal anglais se caractérise par un niveau de grammaticalisation nettement plus avancé que celui d'autres langues germaniques ${ }^{23}$. Par sa fonction grammaticale, le modal saisit anticipativement le lien sujet / prédicat en amont de sa validation, d'où l'absence des marques d'actualisation sur le verbe lui-même (*he may goes, *he can smoked). Par son contenu lexical, chaque modal quantifie la distance séparant le couple sujet / prédicat de la validation prédicationnelle au moment où le locuteur en réalise l'énonciation, ce qui a motivé diverses modélisations de la chronologie notionnelle des modalités que suppose un tel

\footnotetext{
${ }^{22}$ Must a un sémantisme particulier, une pression exercée par le locuteur en vue de la réalisation ou de la reconnaissance de la réalité du procès, laquelle est incompatible avec l'expression d'une prise de recul telle que le passé, qui la neutraliserait.

${ }^{23}$ Jacques François fait remarquer qu'en allemand et en néerlandais on observe des structures en lesquelles le modal occupe la place d'un verbe lexical, d'où des combinaisons telles que zu können, muss können, Das ist gekonnt! « Il y a du savoir-faire là-dedans », "ça c'est un pro ». Le modal anglais, spécialisé qu'il est dans la soudure prédicationnelle entre sujet et prédicat, s'interdit de facto d'instancier le prédicat lui-même et n'admet que la fonction de relateur.
} 
modèle ${ }^{24}$. Le modal suppose que le locuteur ait présélectionné le prédicat visé et s'intéresse désormais au calcul de la relation même, d'où la démission de to $(* \mathrm{MOD}+t o)$, sauf dans le cadre particulier de ought to qui, invoquant une norme de comportement prise pour référence, suppose une pluralité d'énonciateurs comme sources stratifiées de la visée caractéristique de to. Le verbe need a un comportement hybride : suivi de to en contexte positif (He needs to be helped), il se modalise en contexte négatif ou interrogatif (you needn't come), la négation invalidant le ciblage du prédicat ${ }^{25}$.

A l'exception de ce cas très particulier, les modaux sont monophoniques en ce qu'ils attribuent exclusivement au locuteur l'origine de la visée actualisatrice de la prédication, à la différence des périphrases en to, qui rapportent au locuteur la seule sélection du prédicat, et à un autre énonciateur l'origine de la teneur sémantique de la modalité lexicale. Cette simplification par le modal est déterminée par le dépassement de la phase de la sélection du prédicat et la démission de to. Elle permet en outre au système de lexicaliser une donnée supplémentaire : soit le locuteur se pose comme unique responsable du calcul de la modalité (may pour le possible, shall pour la coercition), soit il pose que son choix est motivé par la reconnaissance de propriétés acquises par le référent du sujet (can pour la capacité et le droit acquis, will pour la prédiction) : you shall not kill (you n'est pas porteur des propriétés motivant la prédiction not kill, dont la réalisation implique inévitablement une contrainte), you will not kill (you étant porteur des propriétés motivant la prédiction not kill, il n'y a pas contrainte, mais comportement libre ou naturel de l'agent) ${ }^{26}$. Avec son sémantisme de coercission, must est incompatible avec la reconnaissance de propriétés inhérentes au sujet et n'a pas de contrepartie intrasubjective, d'où l'inexistence d'un sixième modal qui rendrait le système élégamment symétrique mais cognitivement aberrant.

Le contraste qui oppose la monophonie des modaux à la polyphonie des périphrases en to motive deux types de stratégies en cohésion discursive :

1) Cotte 1996 et 1997 a étudié en détail comment une périphrase nominalise et réélabore une modalité en la faisant reprendre par un second énonciateur. Un docteur peut prescrire un médicament à un patient réticent en énonçant you must follow this treatment, propos que l'épouse du malade pourra reprendre par la suite en énonçant you are / have to follow this treatment en justifiant son choix de prédicat par la tierce personne distincte du sujet. Le même phénomène de reprise à caractère polyphonique se produit en cas d'enchâssement de plusieurs modalités : you must come, *you will must come > you will have to come. La modalité will, dont la source est le locuteur, ne peut être appliquée qu'à une modalité imputée à une source distincte, d'où la périphrase avec to, et cette modalité sous-jacente doit être préconstruite, d'où have et jamais be, lequel n'admet aucune forme autre que am / is / are et was / were dans la périphrase verbale (*be to, *being to, *been to), alors que have admet ces constructions. Le recours à la périphrase verbale permet ainsi la résolution de conflits cognitifs engendrés par la confrontation de modalités issues d'instances énonciatives et de niveaux de constructions distincts en syntaxe génétique; plus la superposition de strates est multiple, plus le sujet s'écarte du prédicat dans la transition proposée / transformée de discours avec l'insertion des relateurs de jonction prédicationnelle, avec l'impression que le sens global de l'énoncé ne coïncide pas avec celui des syntagmes de surface :

\footnotetext{
${ }^{24}$ Joly 1978 ; Adamczewski \& Delmas 1982, 47.

${ }^{25}$ Les études sur corpus font apparaître, pour ce verbe ainsi que pour dare « oser », des distributions bien plus complexe, avec notamment des constructions intermédiaires telles que needed / dared + BV. Sur need, cf. De Cornulier 1978.

${ }^{26}$ Les champs de la modalité (possibilité, certitude) sont binarisés en anglais ; les critères d'alternance généralement donnés sont les oppositions extra- / intrasubjectif (Joly 1978), [-/+ inhérent au sujet] (Adamczewski \& Delmas 1982), et modalité forte / faible (Larreya \& Rivière 1993).
} 
I can't seem to get this air conditioner to work. (D. Thomas)

2) inversement, le modal peut être utilisé par le locuteur pour prendre en charge, assumer et actualiser ou valider un choix de modalité antérieurement posé à l'état de puissance par une périphrase verbale en to et rapporté à un énonciateur présupposé. Dans l'exemple qui suit, le père arrose sa fille avec la douche :

(2) She squealed with laughter. 'Daddy, you're not to! Mummy says it's naughty!'

'We mustn't do it then, must we? Hand me the towel, pet.' (J. Braine)

Dans cet exemple transparent, you're not to figure un choix modal puissanciel pour un locuteur qui n'a pas l'autorité de poser un choix effectif, la petite fille, aussi se réfère-t-elle explicitement à la tierce personne invoquée, Mummy; ce choix est ensuite validé sous la forme we mustn't par le père, un locuteur qui a l'autorité requise pour assumer ce choix sans invoquer un destinateur préalable. Ce que montrent ces deux principes de séquencialité adversatifs, c'est qu'il ne faut pas amalgamer la syntaxe génétique (la chronologie cognitive de la construction de la relation prédicative) et la syntaxe résultative (l'ordination énonciative des syntagmes réalisés) : la même modalisation peut figurer dans un contexte successivement en ses états puissanciel et effectif dans un ordre ou dans l'autre, et la cohésion du discours impose que la seconde fasse écho à la première, d'où un effet d'anaphore, qui doit être distingué de l'effet de présupposition lié à la polyphonie des périphrases stratifiées en to,et distingué encore de cet autre effet de présupposition lié aux modaux intrasubjectifs can et will.

\subsection{Do, auxiliaire modalo-grammatical}

La morphologie et la syntaxe de do illustrent sa position intermédiaire dans le système : 1) do se combine à la base verbale non fléchie (modalisation primaire) ; 2) do est une réédition phonologique voisée de to, le premier marqueur de ce champ de la modalité ; 3) do est aussi le premier des trois auxiliaires grammaticaux : do + BV (à accomplir), be + -ing (inaccompli), have $+\mathrm{V}$-ed (accompli). Ceci en fait un auxiliaire modalo-grammatical aux propriétés hybrides motivées par la synthèse des contraintes des deux versants du système. Cette ambivalence se retrouve au plan sémantique : en matière d'aspect, do saisit le procès à accomplir par sa borne inchoative (à la différence de be qui le saisit en intériorité marquée par -ing et have qui le saisit en limite finale marquée par -ed); en matière de modalité, do partage avec to la fonction de ciblage d'une occurrence paradigmatique, mais to cible le procès en opposition à d'autres procès envisageables (cf. supra) et sans liaison à un sujet présélectionné, alors que $d o$, avec sa fonction dialogique polémique, oppose l'actualisation à l'inactualisation, ou le procès à son contraire (même analyse que Culioli ici) : I do speak Basque s'oppose directement et implicitement à I do not speak Basque. Etant donné que do valide l'ensemble de la connexion prédicationnelle, la phase cognitive antérieure marquée par to, celle de la sélection du procès, est dépassée, et le contraste paradigmatique se déplace vers l'alternance en cause lors de la validation de la relation prédicative, à savoir la validité de l'événement référentiel lui-même.

\section{La modalisation secondaire}

La modalisation secondaire consiste pour le locuteur à imposer à l'allocutaire une procédure d'appréhension d'une relation prédicative qu'il considère comme déjà validée. Si la relation préconstruite est appréhendée en cours de validité, la base verbale reçoit la flexion -ing; si 
elle est déclarée périmée, la flexion est -ed (pour un verbe « régulier »). Tous les auxiliaires de visée (modaux et $d o$ ), réservés à la modalisation primaire, sont proscrits puisque la notion même de visée est contre-indiquée par la préconstruction de la soudure prédicationnelle signalée par les flexions -ing et $-e d$. Le système serait parfaitement symétrique, et même redondant, s'il n'existait pas le passif en be $+-e d$ car on aurait $d o+\mathrm{BV}$, be + -ing et have + ed avec homologie parfaite des trois positions respectivement adoptées par les auxiliaires par rapport à l'instant de prédication et l'état dans lequel la prédication est appréhendée par la flexion : do projette à partir du sujet une relation encore non réalisée $(\mathrm{BV})$, be conjoint au sujet une relation en cours de validité (-ing), have disjoint du sujet une relation périmée (-ed). La modalisation secondaire permet au locuteur d'imposer à l'allocutaire un regard appréciatif porté a posteriori sur un procès dont la soudure prédicationnelle est acquise : l'énoncé exige un travail interprétatif qui dépasse la compréhension de l'information explicite. Les configurations fondamentales sont les suivantes :

\section{1. $B e+-$-ing}

Cette périphrase articule systématiquement un symptôme à un diagnostic, avec deux cas de figure : 1) soit l'énoncé en be + -ing exprime le symptôme et implicite le diagnostic que le locuteur invite l'allocutaire à établir : Daddy's reading the newspaper ne fait pas sens par sa valeur descriptive (quel intérêt y a-t-il à décrire par des mots une situation visuelle sur laquelle les colocuteurs sont renseignés par perception sensorielle immédiate ?) mais par son implication pragmatique, que le contexte seul permet de déterminer (il est étonnant que papa lise pendant le match de football, ou cet énoncé vient en réponse à une question de maman qui s'étonne de ne pas trouver papa en un lieu précis à un moment donné, ou encore l'énoncé invite un enfant à ne pas déranger son père, etc.). Le procès anaphorisé par la prédication préconstruite est pris pour emblême de la propriété à construire et que be attribue au sujet, en l'occurrence «le comportement constaté de papa est anormal ou surprenant et appelle une analyse approfondie ». 2) soit l'énoncé en be + -ing reformule une situation prise pour symptôme et antérieurement présentée par un prédicat sans -ing, auquel cas be + -ing explicite cette fois le diagnostic lui-même : when a twenty-year-old woman marries an eightyyear-old man, she is marrying money. Money vient se substituer à l'ancien objet de marry, ce qui suppose la préconstruction marrying, et -ing déclenche la correction des propriétés de woman : elle est intéressée. Cette préconstruction est valable pour toutes les structures suivies de -ing : it began raining laisse entendre " conformément à ce que la situation laissait prévoir ou à ce qui avait été annoncé ", par opposition à it began to rain, purement constatif; she loves cooking fait du jugement love un diagnostic déduit de l'observation d'un fait présupposé - une modalisation secondaire - par opposition à she loves to cook, principe de comportement (modalisation primaire) déterminant pourquoi, par exemple, elle ne fréquente jamais les restaurants. La valeur aspectuelle durative de la périphrase "continue » be $+-i n g$, que la morphosyntaxe superficielle semble appliquer au verbe du prédicat et faire porter sur le référent événementiel extraliguistique, concerne en réalité le temps cognitif de la construction de la prédication que le locuteur invite l'allocutaire à consacrer à l'interprétation du message ; l'instruction métalinguistique impliquée par cette récupération modale d'un système emprunté à la catégorie de 1 'aspect ${ }^{27}$ est « prenez le temps de méditer le sens du procès que je vous soumets et tirez-en les conclusions qui s'imposent», la durée du procès exprimé servant métaphoriquement de vecteur à celle de l'interprétation « imprimée ».

\footnotetext{
${ }^{27}$ Robert 1994.
} 


\section{2. $B e+-e d$}

L'ordre des syntagmes nominaux dans l'énoncé transitif est régi par deux principes de distribution linéaire dont l'action peut être harmonieuse ou conflictuelle: 1) par son sémantisme relationnel, le verbe, centre organisateur de la matrice actancielle des arguments, attribue le rôle d'agent à l'argument « gauche » et celui de « patient » à l'argument « droit » : AGENT < eat > PATIENT. 2) le discours, centre organisateur de la répartition des topiques et des focales, attribue à la partie gauche de l'énoncé le statut mémoriel (par adhésion iconique du premier syntagme nominal énoncé au déjà dit antérieur) et le statut amémoriel à la partie droite. Le second principe est dominant et le premier dominé : s'il se trouve que le verbe distribue l'agent et le patient de la même manière que la cohésion discursive répartit topique et focale, alors le locuteur se borne à laisser constater à cette adéquation non problématique sans mot dire et laisse passer la prédication sans la mentionner, cats eat mice. Mais il se rencontre aussi le conflit cognitif par lequel la cohésion discursive thématise le patient plutôt que l'agent, l'installant dans la position opposée à celle que prescrit la matrice actancielle du verbe à ses arguments. La résolution du conflit cognitif passe par 1) la neutralisation de la capacité du verbe à imposer un principe de distribution actancielle à ses arguments : -ed (participe passé), forme de péremption de la prédication, coupe le verbe de ses arguments collatéraux ; le verbe cesse ainsi d'imposer une matrice argumentale d'orientation inverse à l'échelonnement informationnel du connu vers l'inconnu. 2) la reconstruction par des moyens ad hoc d'une matrice argumentale inverse, d'orientation mise en conformité à celle de la cohésion discursive : be, d'aspect statif, fait du sujet le site du procès et non son vecteur, le rendant compatible avec le rôle de patient non dynamique ; et, le cas échéant, by, préposition instrumentale à valeur cinétique, fait de son complément un support vectoriel compatible avec la notion d'agentivité : mice are eaten by cats. Le type de modalisation périphrastique réalisé par le passif est purement structural et métalinguistique: il constitue la réponse grammaticalisée à la détection du conflit cognitif suscité par la collision de principes de distribution contradictoires des arguments nominaux, l'un subordonné émanant d'un constituant intégré par l'énoncé, un mot, en l'occurrence le verbe ; l'autre, hyperordonné, émanant du constituant intégrant l'énoncé, le discours. Il y a modalisation en ce que la détection par le locuteur d'un conflit cognitif entre deux principes d'ordination sémantique appelle la grammaticalisation d'une réponse préventive destinée à l'allocutaire, lequel ne peut être livré à la gestion de la contradiction sans « notice de montage » métalinguistique de la procédure résolutoire.

\subsection{Have + -ed}

A l'inverse de be intransitif, have est lui-même transitif, si bien que si le verbe l'est également sa matrice actancielle est préservée. De ce fait le rôle de $-e d$ n'est pas de la neutraliser mais de signaler l'écart entre le moment où se construit la propriété du sujet (he ate too much) et le moment où le locuteur la fait constater par l'allocutaire (he has eaten too much), avec un décalage entre deux instances référencielles passée et présente du signifié du sujet : l'absence d'auxiliaire entre he et ate adhère à l'absence d'écart entre l'agent et le procès, donc le référent de he dont il est parlé à l'instant d'énonciation est en réalité passé, et la jointure prédicationnelle non filtrée par have laisse le morphème verbal -ed de péremption affecter le sujet, dont on n'envisage qu'un référent obsolète dont on ignore même s'il existe encore. Inversement, have pose un écart entre le sujet he, support de prédication à l'instant de parole, et la prédication eat déclarée périmée : l'auxiliaire entérine la désynchronisation des référents du sujet (présent, ce qu'indique have) et de l'événement (passé, ce qu'indique $-e d$ ). Ceci recoupe l'analyse d'Adamczewski, selon qui « have porte au crédit du sujet » l'acquis d'une 
connexion prédicationnelle préconstruite au prétérit, ce qu'il glose John has [John bought a car] ; cette analyse illustre également la théorie guillaumienne de l'incidence du verbe au sujet, puisqu'elle suppose un transfert au sujet du trait passé du verbe (he ate) ou présent de l'auxiliaire (he has eaten) en vue de conduire la référenciation pertinente alors même que ce sujet ne porte en lui-même aucun indice temporel différenciateur.

\section{Conclusion}

Les périphrases verbales sont des locutions verbales plus ou moins complexes destinées au traitement de la modalisation primaire ou secondaire de la relation prédicative en syntaxe génétique. Elle sont liées à d'autres structures de prédication par cette problématique, comme les verbes aspectuels ou les verbes de relation intersujets. Elles constituent des préconstruits délexicalisés, plus ou moins grammaticalisés, avec des valeurs pragmatiques stabilisées. Il existe donc une systématique de la modalisation et de la construction de la prédication qui sert de dénominateur commun. Il semble que d'un point de vue guillaumien on peut difficilement parler de chronogenèse du verbe anglais, mais on peut envisager une procédure de traitement de la prédication dans l'ordre ici proposé et dont la phase centrale est le pivot et la forme par défaut. Ce système est d'un type très différent de celui des autres langues germaniques.

\section{Bibliographie}

ADAMCZEWSKI, Henri \& DELMAS, Claude (1982), Grammaire Linguistique de l'Anglais, Colin.

BOTTINEAU, Didier (1999), Aspect, actance et modalité : systématique de l'infinitif anglais, thèse, Paris IV.

- (2001), «To entre aspect, actance et modalité », de CARVALHO, Paulo, QUAYLE, Nigel, ROSIER, Laurence, SOUTET, Olivier (éds.), La psychomécanique aujourd'hui, Actres du $8^{e}$ Colloque international de psychomécanique du langage. Seyssel. 1997, Honoré Champion, Paris, France, 49-84.

- (2002a), «Les cognèmes de l'anglais : principes théoriques », LOWE, Ronald (éd.), en collaboration avec PATTEE, Joseph et TREMBLAY, Renée, Le système des parties $d u$ discours, Sémantique et syntaxe, Actes du IX colloque de l'Association internationale de psychomécanique du langage, Les Presses de l'Université Laval, Québec, Canada, 423-437.

- (2002b), «Sémantique et morphosyntaxe des verbes aspectuels », DELMAS, Claude, ROUX, Louis (éds.), Construire \& Reconstruire en linguistique anglaise, Syntaxe et sémantique, C.I.E.R.E.C. Travaux 107, Publications de 1'Université de SaintEtienne, France, 209-242.

- (2003), «Les cognèmes de l'anglais et autres langues », OUATTARA, Aboubakar (éd.), Parcours énonciatifs et parcours interprétatifs, Théories et applications, Actes $d u$ Colloque de Tromsø organisé par le Département de Français de l'Université, 2628 octobre 2000, Ophrys, Gap, France, 185-201.

- (2004a), «Correction normative, correction cognitive : le split infinitive », ROUX, L. \& DELMAS, C., C.I.E.R.E.C. Travaux, CXIII, p. 25-48.

- (2004b), « Le problème de la négation et sa solution dans la langue anglaise : le cognème $\mathrm{N}$ », DELMAS, Claude \& ROUX, Louis (dir.), La contradiction en anglais, C.I.E.R.E.C. Travaux 116, Publications de l'Université de Saint-Etienne, 27-53. 
- (2004c), «Les cognèmes $\mathrm{S}$ et $\mathrm{T}$ en langue anglaise, ANGOUJARD, Jean-Pierre, BOTTINEAU, Didier, WAUQUIER, Sophie (éds.), Universaux Sonores, Actes des $3^{e}$ Journées d'Etudes Linguistiques, Université de Nantes, 21-23 mars 2002, Presses Universitaires de Rennes, France, sous presse.

CHUQUET, Jean (1986), «TO et l'infinitif », Cahiers de recherche en grammaire anglaise, numéro spécial, Ophrys, Gap.

COTTE, Pierre (1982a), "Autour de TO », in Travaux du CIEREC, XXXV, Université de Saint-Etienne, p.57-80.

- (1982b), «TO, opérateur de dévirtualisation en anglais », Modèles Linguistiques, IV,2, Presses Universitaires de Lille, p.135-149.

- (1983), «Forme et fonctionnement en grammaire anglaise » in Travaux XXXIX, CIEREC, Saint-Etienne, 7-32.

- (1992), « Réflexions sur la linéarité » Travaux LXXVI, CIEREC, Saint-Etienne, 53-76.

- (1996), L'explication grammaticale de textes anglais, P.U.F.

- (1997), Grammaire linguistique, Didier-Erudition.

DE CORNULIER, Benoît (1978), « Spécialisation pragmatique de la construction auxiliaire du verbe anglais need» in Stratégies discursives, Presses Universitaires de Lyon, Actes du Colloque du Centre de Recherches Linguistiques et Sémiologiques de Lyon, 20-22 mai 1977, 123-32.

DELMAS, Claude (1987), Structuration abstraite et chaîne linéaire en anglais contemporain, Klincksieck.

DOUAY, Catherine (2000), Eléments pour une théorie de l'interlocution, Presses Universitaires de Rennes.

DOUAY, C. \& ROULLAND, D. (1996), «L'absence de marque verbale en anglais dans une théorie de l'interlocution », Absence de marques et représentations de l'absence (1), Travaux linguistiques du CERLICO, 9, 311-326.

DUFFLEY, Patrick (1992), The English Infinitive, Longman.

GUILLAUME, G. ([1929] 1965), Temps et verbe. Théorie des aspects, des modes et des temps, Champion, Paris.

- (1964), Langage et science du langage, Les Presses de l'université Laval, Québec; Nizet, Paris.

- (1971-98), Leçons de linguistique, 16 vol., Les Presses de l'université Laval, Québec; Klincksieck, Paris; Presses Universitaires de Lille.

JOLY, A. (1978), " Esquisse du système des modaux en anglais contemporain », Explorations linguistiques et stylistiques, CIEREC, Travaux XXII, Saint-Etienne, 83-95.

JOLY, André \& O'KELLY, Dairine (1990), Grammaire systématique de l'anglais, Nathan, Paris.

POTTIER, Bernard (1992), Sémantique générale, P.U.F.

QUAYLE, Nigel (1996), "Pour une valeur fondamentale du $-s$ en anglais », Modèles linguistiques, 33, 165-176.

ROBERT, Stéphane (1994), "Sur le rôle du sujet énonciateur dans la construction du sens : liens entre temps, aspect et modalité », Subjecthood and Subjectivity, The Status of the Subject in Linguistic Theory, YAGUELLO, Marina (ed), Ophrys, Gap, 209-229.

ROULLAND, Daniel (1992), "Sur la subordination non finie en anglais contemporain », Travaux linguistiques du CERLICO, 5, Subordination subordinations, Rennes, 158-84.

VALIN, Roch (1981), Perspectives psychomécaniques sur la syntaxe, Les Presses de l'université Laval, Québec. 